
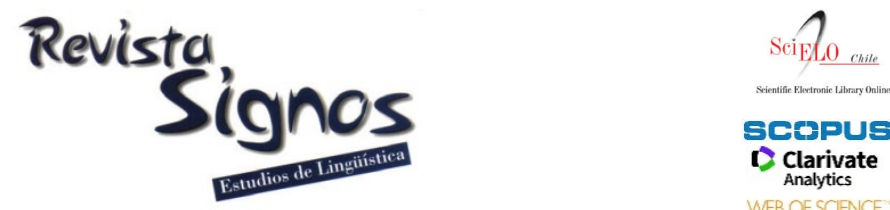

\title{
Los recursos y la dinámica evaluativa en español: El discurso de la memoria colectiva en la Argentina postdictatorial
}

\section{Evaluative resources and dynamics in Spanish: The discourse of collective memory in post-dictatorship Argentina}

\author{
Mariana Pascual \\ PONTIFICIA UNIVERSIDAD CATÓLICA DE CHILE \\ CHILE \\ mariana.pascual@uc.cl
}

Recibido: 25-IX-2019 / Aceptado: 23-X-2020

DOI: $10.4067 /$ S0718-09342021000100258

\section{Resumen}

Los estudios de los significados interpersonales han cobrado gran auge desde el desarrollo del Sistema de Valoración (Martin, 2000, 2002; Martin \& White, 2005; Hood \& Martin, 2005) en el marco de la Lingüística Sistémico Funcional (Halliday, 1978; Halliday \& Matthiessen, 2014). Si bien este marco fue inicialmente postulado para el análisis de cuerpos de datos en inglés, su aplicación se ha extendido notablemente y ha demostrado resultar de utilidad en una amplia variedad de lenguas. Este trabajo tiene como objetivo principal presentar una propuesta de los recursos léxico-gramaticales que construyen significados evaluativos en español. Asimismo, establece una correlación entre el tipo de reacción social de la Argentina en las dos décadas que siguieron a la dictadura militar (1974-1983) y un desarrollo sistemático gradual de los significados valorativos, que denominamos Dinámica Evaluativa (DE). La propuesta deriva del análisis del discurso de medios argentinos en el período 1984-2004 y los resultados dan cuenta de una mutación en la construcción de Juicios, tanto de sanción como de estima social, ya que a medida que se aleja el hecho social traumático de la dictadura, se generan combinaciones de significados que incorporan elementos de Afecto, los que se fusionan para re-presentar cómo la sociedad enfrentó su pasado reciente y desarrolló su memoria colectiva. En línea con estos hallazgos, se pudo observar que la predominancia de Juicios explícitos dio lugar a lo largo de los años a valores de Afecto explícito que invocan Juicios. Los datos analizados permitieron sistematizar los recursos valorativos utilizados y proponer un glosario de lexemas relacionados con las dimensiones evaluativas de los discursos sobre pasados traumáticos. La propuesta puede resultar de utilidad para identificar, sistematizar e interpretar la construcción discursiva de reacciones sociales a fenómenos de profundo impacto traumático, como las guerras civiles, dictaduras, invasiones, enfrentamientos religiosos y muchos otros conflictos que dejan profundas huellas de dolor en nuestras sociedades. 
Palabras Clave: Sistema de la Valoración, recursos evaluativos, Dinámica Evaluativa (DE), pasado reciente, memoria colectiva.

\begin{abstract}
Studies focusing on interpersonal meanings have attracted the attention of many scholars since the development of the System of Appraisal (Martin, 2000, 2002; Martin \& White, 2005; Hood \& Martin, 2005) in the context of Systemic Functional Linguistics (Halliday, 1978; Halliday \& Matthiessen, 2014). Though initially applied to the study of data in English, its application has widened considerably, proving useful for the analysis of discourse in a variety of languages. This study aims at presenting a proposal related to the lexicogrammatical resources that construe evaluative meanings in Spanish. It also establishes a correlation between the type of social reaction that took place in postdictatorship Argentina (1974-1983) and a gradual, systematic development of appraisal meanings, which we define as Evaluative Dynamics (ED). The proposal derives from the analysis of Argentinian media discourse from the period 1984-2004, and the results indicate a diachronic transformation in the construction of values related to Judgement -social sanction as well as social esteem- which turn into and combine with values that are more closely related to the dimension of Affect. It seems that meanings of Judgement and Affect fuse in order to represent how Argentina faced its recent past and developed a collective memory. In line with these findings, it was observed that the clear dominance of explicit judgement values gradually gave rise to values of explicit Affect which, at the same time, evoke Judgement. The data analyzed allowed for the systematization of resources identified and for the proposal of a glossary of terms related to the evaluative dimension of discourses on traumatic pasts, collective memory and recent histories. The proposal may prove useful for the identification, systematization and interpretation of the discoursal construction of social reaction to traumatic phenomena, as after civil wars, military dictatorships, invasions, religious conflicts, forced immigrations and various other social conflicts that leave profound wounds in our societies.
\end{abstract}

Key Words: System of Appraisal, evaluative resources, Evaluative Dynamics (ED), recent past, collective memory.

\title{
INTRODUCCIÓN
}

De la misma manera que las vidas de los individuos se ven marcadas por la partida de seres queridos, rupturas de relaciones, crisis identitarias y muchas otras situaciones traumáticas, las sociedades atraviesan momentos de profundas crisis. En línea con lo que sucede en la esfera personal de los conflictos, las sociedades enfrentan una variedad de posibilidades para seguir adelante luego del trauma. Algunas veces las opciones adoptadas son conscientes, otras no. Algunas son consideradas como 'saludables' o 'curativas', otras como estancamientos y frustraciones por no poder superar el trauma y asumir una nueva realidad (Assmann, 2009). Este trabajo hace referencia a una de estas situaciones que se desencadenan con posterioridad a una crisis, en particular, a la manera en que la sociedad argentina reaccionó ante el enorme trauma que significó la dictadura militar que padeció entre 1974 y 1983. 
Para aproximarnos a este objeto de estudio desde una perspectiva discursiva, recurrimos a una herramienta analítico-interpretativa de suma utilidad: el análisis sistemático de los significados interpersonales, definidos como aquellos que son instanciados en los textos por medio de los recursos "relativos a la negociación de las relaciones sociales: cómo las personas interactúan, incluyendo los sentimientos que experimentan y comparten" (Martin \& Rose, 2008: 22). Los estudios de los significados interpersonales han atraído gran atención desde el desarrollo del Sistema de Valoración (Martin, 2000, 2002; Martin \& White, 2005; Hood \& Martin, 2005) en el marco de la Lingüística Sistémico Funcional (Halliday, 1978; Halliday \& Matthiessen, 2014). Si bien este marco fue inicialmente postulado para el análisis de cuerpos de datos en inglés, su aplicación se ha extendido notablemente y ha demostrado resultar de utilidad en una amplia variedad de lenguas.

Fairclough (2010) afirma que existe una correlación entre la dinámica de las prácticas sociales y el desarrollo de ciertas configuraciones que surgen del uso sistemático de los recursos lingüísticos que construyen significados evaluativos. Esta propuesta de correlación entre lo social y lo semántico-discursivo como parte constitutiva y determinante es consistente con los postulados básicos de la LSF y de los enfoques críticos del discurso (Wodak, 1995, 1996, 2013; Wodak \& Meyer, 2009).

Siguiendo esta propuesta, desarrollamos este trabajo, en el que planteamos un estudio diacrónico del tipo de reacción social de la Argentina a lo largo de las dos décadas que siguieron a la dictadura militar y, por otra parte, realizamos indagaciones que determinan un desarrollo sistemático gradual de los significados valorativos. A este fenómeno discursivo lo denominamos Dinámica Evaluativa (DE) (Pascual, 2014, 1017a, 2017b). A tal fin, conformamos un corpus constituido por un total de 60 artículos publicados entre 1984 y 2004, escogidos de seis periódicos de diferentes zonas de la Argentina.

Los textos fueron analizados manualmente, sin el auxilio de software especializado, para la identificación de significados evaluativos explícitos e implícitos, siguiendo la propuesta del Sistema de la Valoración (Martin, 2000, 2002; Martin \& White, 2005; Hood \& Martin, 2005). Este sistema propone tres dominios semántico-discursivos generales: COMPROMISO, ACTITUD y GRADACIÓN. En este trabajo nos focalizaremos en los significados actitudinales, que, a su vez, se subdividen en Afecto -la expresión de los sentimientos-, Juicio -nuestras opiniones sobre los otros y sus acciones- y Apreciación -las evaluaciones sobre el valor social, estético de los objetos y valores del mundo.

$\mathrm{Si}$ bien numerosos estudios previos ofrecen acercamientos a la temática de la creación y desarrollo de la memoria colectiva, no se conocen a la fecha estudios que analicen la construcción de este fenómeno social desde una perspectiva discursiva, sobre la base de los recursos léxico-gramaticales. Nuestro recorrido por la bibliografía 
tampoco arrojó trabajos que analizaran corpus de dimensiones considerables y de representatividad diacrónica, que dieran cuenta de manera sistemática de los recursos léxico-gramaticales que son específicos en la construcción de la memoria de los pasados traumáticos recientes.

En consecuencia, nos proponemos en este estudio un doble propósito. Primeramente, proponer una tipología de recursos léxico-gramaticales que construyen significados evaluativos en español para la construcción discursiva de los pasados traumáticos y, a su vez, establecer una correlación entre el tipo de reacción social de la Argentina en las dos décadas que siguieron a la dictadura militar (1974-1983) y un desarrollo sistemático gradual de los significados valorativos, su Dinámica Evaluativa (DE).

\section{Marco teórico}

\subsection{El género noticias en el marco de los estudios sistémico- funcionales}

Adoptamos aquí marcos teórico-metodológicos vinculados de diversas maneras al análisis de problemáticas sociales sensibles, que postulan un acercamiento discursivo a los textos y que se basan en la convicción del aporte disciplinar a la resolución de problemáticas sociales. Como marco general, nos situamos en el contexto de la Lingüística Sistémico Funcional (LSF) (Halliday, 1978; Halliday \& Matthiessen, 2014). La Tabla 1 muestra las dimensiones del lenguaje desde esta perspectiva. Nuestro foco será en la dimensión del sistema, en tanto analizaremos las opciones paradigmáticas realizadas por los escritores de las noticias, en el principio de instanciación y en el orden de la léxico-gramática, aunque, tal como lo postula la teoría, las dimensiones son simultáneas y complementarias.

Tabla 1. Las dimensiones del lenguaje y sus principios ordenadores (Halliday \& Matthiessen, 2014: 20).

\begin{tabular}{|l|l|l|}
\hline \multicolumn{1}{|c|}{ Dimensión } & \multicolumn{1}{|c|}{ Principio } \\
\hline $\begin{array}{l}\text { estructura } \\
\text { (orden sintagmático) }\end{array}$ & rango & $\begin{array}{l}\text { Órdenes } \\
\text { clausula } \sim \text { grupo } / \text { frase } \sim \text { palabra } \sim \text { morfema } \sim \\
\text { grupo tonal } \sim \text { pie } \sim \text { sílaba } \sim \text { fonema } \sim \text { fonología } \sim\end{array}$ \\
\hline $\begin{array}{l}\text { sistema } \\
\text { (orden paradigmático) }\end{array}$ & delicadeza & gramática $\sim$ léxico $\sim$ léxico-gramática ; \\
\hline estratificación & realización & semántica $\sim$ léxico-gramática $\sim$ fonología $\sim$ fonética \\
\hline instanciación & instanciación & $\begin{array}{l}\text { potencial } \sim \text { subpotencial } / \text { tipo de instancia } \sim \\
\text { instancia }\end{array}$ \\
\hline metafunción & $\begin{array}{l}\text { ideacional lógica [experiencial } \sim \text { interpersonal] } ~ \\
\text { textual }\end{array}$ \\
\hline
\end{tabular}

En particular, este trabajo abreva en la propuesta de la Escuela de Sídney en lo que respecta a la Teoría de Géneros (Martin \& Rose, 2008: 5), definidos como 
"configuraciones recurrentes de significados que materializan las prácticas sociales de una cultura dada". Esta definición resulta de particular aplicabilidad para el tipo de discurso que aquí presentamos, el discurso de los medios. En este sentido, aplicamos la clasificación presentada por Feez, Iedema y White (2008) para los géneros mediáticos que estos autores proponen (Figura 1), que incluye tres tipos generales de géneros para los discursos mediáticos 1) géneros de historias (story genres), 2) géneros factuales y 3) géneros de críticas.

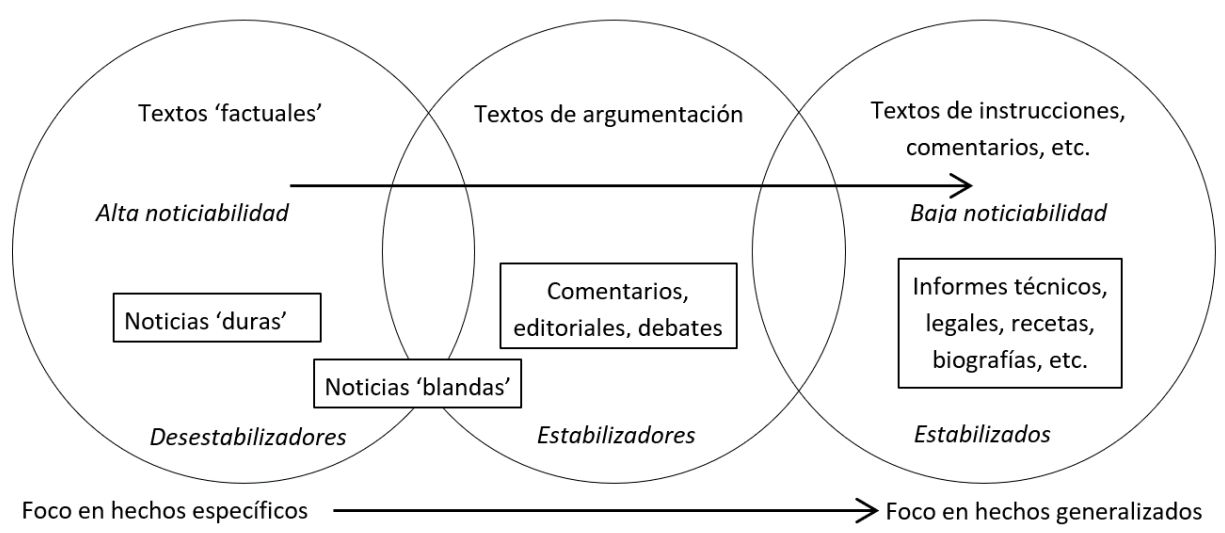

Figura 1. Tipos de textos de los medios (Feez, Iedema \& White, 2008: 68).

Esta propuesta resulta de particular utilidad por considerar múltiples variables, tales como el propósito social del discurso (ej. argumentar, dar instrucciones), el índice de noticiabilidad (alta, media o baja), el impacto social del género (desestabilizador, estabilizador o estabilizado), y combina estos criterios con las denominaciones cotidianas de los géneros (tales como 'comentarios', 'debates', 'recetas'). A la vez, establece una correlación con el foco de los hechos que se reportan, ya sea hechos específicos, como en el caso de las noticias 'duras' que refieren a accidentes, desastres naturales, atentados, y otros fenómenos de alto impacto mediático y los géneros de foco en hechos generalizados, de carácter más atemporal, que suelen acompañar y complementar de modo secundario a los textos de alto impacto (como recetas, horóscopos, biografías, entre muchos otros). Los textos seleccionados para este trabajo pertenecen al género noticias blandas, definidos como textos con características tanto de estabilización social como generadores de desestabilización, en tanto combinan textos 'factuales', sobre eventos tales como demostraciones populares o resultados de juicios con textos de argumentación, como los publicados en conmemoración de las víctimas del terrorismo de estado en Argentina durante el gobierno militar.

\subsection{El Sistema de la Valoración}

En un nivel más específico de análisis, seguimos la propuesta del Sistema de la Valoración que Martin y sus colaboradores han desarrollado a lo largo de las dos 
últimas décadas (Martin, 2000, 2002; Martin \& White, 2005; Hood, 2005; White, 2002, 2003, entre muchos otros). Si bien este sistema ha sido denominado 'teoría' por diversos autores, Martin mismo (2016) hace referencia a la necesidad de denominarlo 'sistema', en tanto constituye en desarrollo teórico analítico de un tipo de metafunción en particular, la interpersonal, en el marco de la Teoría Sistémico Funcional, del mismo orden que los sistemas de 'transitividad' en el nivel de significados experienciales, el de 'Tema y rema' en la textualidad, entre otros. Este sistema elabora la noción de significado interpersonal; explora, describe y explica el modo en el que el lenguaje se usa para evaluar, adoptar posiciones, construir personas textuales y manejar posturas y relaciones interpersonales. Se ocupa de la expresión lingüística de la actitud y la emoción. La Figura 2 presenta una versión abreviada del Sistema de Valoración, que no será descripto en profundidad en este trabajo por escapar a los propósitos y límites de este capítulo.

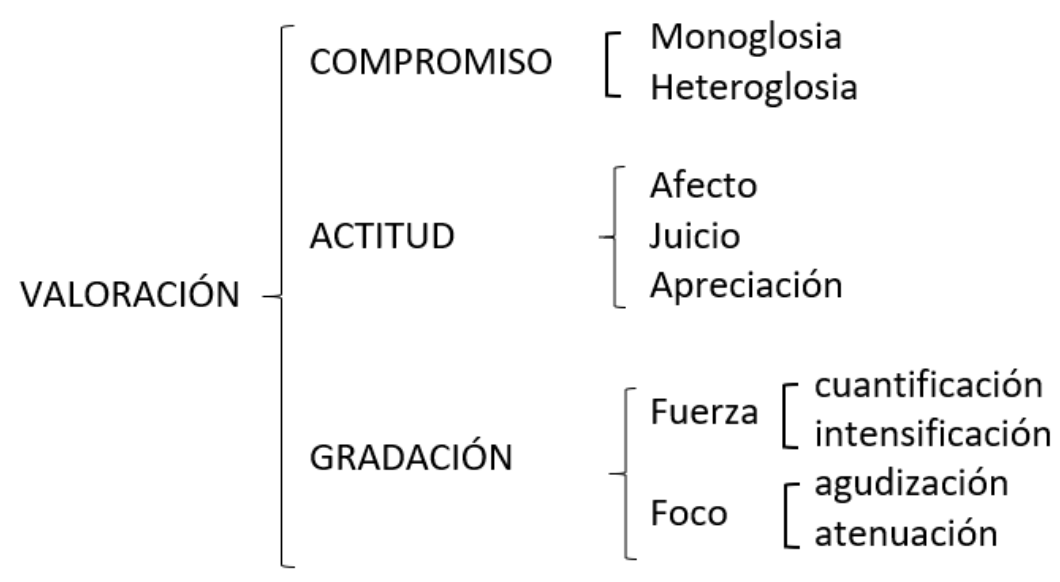

Figura 2. El Sistema de la Valoración (adaptado de Martin \& White, 2005).

Debemos aclarar que en este trabajo nos focalizaremos exclusivamente en el Sistema de ACTITUD, que constituye un dominio semántico-discursivo dividido en Afecto -la expresión de los sentimientos-, Juicio - nuestras opiniones sobre los otros y sus acciones- y Apreciación -las evaluaciones sobre el valor social, estético de los objetos y valores del mundo. Sin embargo, resulta relevante destacar que los recursos léxico-gramaticales operan discursivamente para la creación de múltiples capas de significado y este es un concepto clave en el desarrollo de nuestra propuesta, en la implementación de nuestro análisis y en la lógica que nos guía en la interpretación de los resultados obtenidos.

\subsection{El Enfoque Histórico Discursivo (EHD), memoria y olvido}

En el generoso panorama de enfoques modernos al análisis de los discursos, el papel de las escuelas críticas (Fairclough, 2010; van Dijk, 2008) es indudable. A su vez, 
existe una amplia variedad de miradas críticas, entre las que se destaca el Enfoque Sociohistórico discursivo (EHD) propuesto principalmente por Wodak (1995, 1996, 2007) y Reisigl y Wodak (2001). Se destaca por sus indagaciones sensibles, con un fuerte anclaje textual, y por una incorporación sistemática de los factores históricos en la interpretación de los discursos. Resulta de particular utilidad en el análisis de fenómenos como el que proponemos, en gran medida determinados e interpretables solo a la luz de los factores sociohistóricos que los condicionaron.

El EHD enfatiza fundamentalmente el contexto histórico de producción textual. $\mathrm{Al}$ analizar los discursos relativos a una problemática a lo largo de un período de tiempo, de manera diacrónica, puede develar tendencias en cuanto a los modos en que la argumentación cambia, tornándose más abierta o cerrada. Algunos de los interrogantes que este enfoque plantea hacen referencia a las instituciones sociales, cuestionando sus niveles de inclusión y tendencias a la equidad. De igual modo, este enfoque resulta de utilidad al tratar de determinar si un período o lugar histórico se caracteriza por diferentes niveles de comunicación sistemáticamente distorsionada (Wodak, 2007).

En el marco de este enfoque se han realizado estudios de enorme valor, entre los que destacan los estudios de la construcción discursiva de la memoria colectiva en contextos sociales postraumáticos, tales como los de Assman (2009). Esta autora realiza un aporte singular al analizar los tipos de reacción de diversas sociedades en situaciones post-traumáticas, como lo son las guerras, desastres naturales, genocidios y otros flagelos que dejan profundas huellas en el tejido social.

Fenómenos sociales de características similares y desde el contexto latinoamericano han sido analizados, por ejemplo, desde de los estudios de memoria y pasado reciente de Achugar (2008) sobre la dictadura en Uruguay o los aportes de Oteíza (2010) sobre las representaciones de historia reciente de Chile bajo la dictadura en textos escolares. Sin embargo, estos trabajos no tienen como foco de indagación el carácter dinámico de la construcción de la memoria social.

Para el EHD el análisis del discurso se convierte en una herramienta necesaria e inevitable para el análisis de la evolución de la sociedad y para la crítica de las representaciones de los procesos de comunicación como el que se presenta aquí.

\section{Metodología y corpus}

\subsection{Sobre el período analizado}

El período analizado en este trabajo inicia en 1984, año inmediatamente posterior al retorno a la democracia en Argentina, luego de siete años de una dictadura militar que es reconocida como el período de mayor crueldad y violencia que haya atravesado el país en su historia. El golpe de estado tuvo lugar el 24 de marzo de 1976 y los 
militares se mantuvieron en el poder hasta diciembre de 1983, cuando Raúl Alfonsín fue elegido presidente por la vía democrática. La dictadura militar dejó un saldo lamentable de varios miles de desaparecidos, se debate hasta en la actualidad el número, que varía entre nueve mil y treinta mil. Fue una etapa de heridas profundas, caracterizada por la privación ilegal de la libertad, por la suspensión del estado de derecho, la venta de bebés nacidos en cautiverio y un despliegue de herramientas de coacción y ejercicio de la violencia sin precedentes en nuestra historia.

En virtud de que nuestro foco de atención es la construcción de la memoria, analizamos artículos de diarios publicados a lo largo de dos décadas, desde 1984 y hasta 2004, año de la creación del Museo de la Memoria en el edificio en el que funcionó uno de los más terribles centros de detención y tortura durante la dictadura. Durante esos 20 años Argentina estuvo bajo diez presidencias. Esto se explica ya que algunos mandatarios perduraron en el poder durante 10 años, como es el caso de expresidente Carlos Menem, mientras que otros duraron escasamente una semana, en situaciones de crisis institucionales muy profundas (Benítez \& Mónaco, 2007).

\subsection{Sobre los criterios de selección de textos y la composición del corpus}

La selección se basó en una multiplicidad de criterios, que incluían a) una clara configuración de significados que construyeran el mismo género discursivo, en tanto los medios incluyen gran variedad de géneros (presentados en el Gráfico 2) pero se optó por incorporar solamente aquellos textos que pertenecieran al género 'noticias blandas' o de interés humano (Feez et al., 2008); b) la estricta relación de tópico de los artículos, ya que todos debían hacer referencia a las asociaciones defensoras de derechos humanos, en particular a Madres. La contemplación estricta de este criterio nos permitió recuperar una referencia de manera consistente a la asociación, para poder construir el análisis de la dinámica de Madres de Plaza de Mayo a lo largo del tiempo.; c) simultaneidad de publicación, entre otros. Así, el corpus incluyó un total de 60 noticias blandas publicadas por seis medios en diez fechas distribuidas a lo largo de dos décadas.

Asimismo, se tuvieron en cuenta criterios de distribución regional, procurando que las diversas zonas del país estuviesen representadas. Se seleccionaron seis diarios de diversas regiones, que se presentan en la Figura 3. 


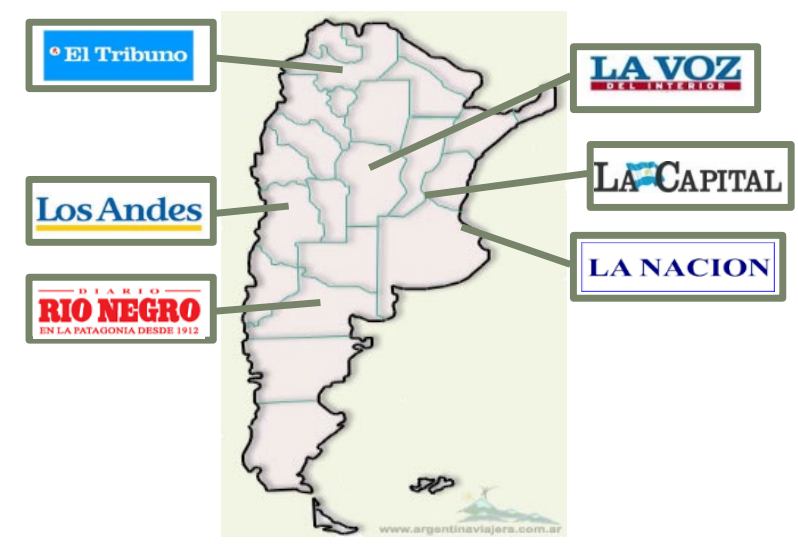

Figura 3. Medios seleccionados y lugar de publicación.

Los 60 artículos fueron publicados en los 6 diarios de mayor influencia en su área. Se observó que los artículos fuesen publicados en el cuerpo principal de los diarios, no en suplementos. Todos los textos correspondían a las características de género y subgénero propuestas por Feez et al. (2008), reconocidas como noticias del subtipo de interés humano.

En todos los casos los artículos hacían referencia al tópico de la dictadura, la memoria y el olvido, en el titular, en el cuerpo de la noticia o en cualquiera de sus componentes. Se excluyeron noticias breves en tanto un análisis exploratorio dio cuenta que no presentaban material rico para el análisis de formas evaluativas. Se estableció un criterio de un mínimo de 100 palabras por artículo. En casos de multiplicidad de textos publicados en igual fecha, se priorizó la noticia local. La totalidad de los textos del corpus principal fueron elegidos por su referencia a tópicos representados en todos los medios y en una fecha idéntica o muy aproximada.

De este modo, el corpus quedó conformado de la siguiente manera: un total de 60 noticias, diez publicadas en cada uno de los diarios analizados: El Tribuno (Salta), Los Andes (Mendoza), La Capital (Rosario), La Nación (Buenos Aires), La Voz del Interior (Córdoba) y Río Negro (General Roca). El total de palabras del corpus es de 28904, distribuidas en 976 complejos clausales.

\subsection{Análisis: El Sistema de la Valoración y la Dinámica Evaluativa}

Se llevó a cabo un análisis manual de todos los textos, identificando las instancias de ACTITUD, que fueron aisladas para su posterior clasificación en términos de recursos léxico-gramaticales y posterior interpretación de la densidad evaluativa.

En lo que respecta a la identificación de recursos, se señalaron no solamente las explícitas sino también las implícitas, en consonancia con lo propuesto por la teoría (Martin \& White, 2005). Asimismo, se consideró que la evaluación se construye no 
solamente a partir de la codificación inscripta (por medio de adjetivos, adverbios, adjuntos modales, fundidas en procesos, entre otros) sino también en formas invocadas mediante provocaciones, en las cuales los significados experienciales son suficientes para invocar la valoración o de invitaciones, instancias en las que existe algún indicio a nivel explícito lexical que le señalan al lector el tipo de evaluación que se plantea. Las formas invocadas son reconstruidas en gran medida a partir del conocimiento del contexto.

En lo que respecta a la dinámica evaluativa (DE), se realizó una comparación cronológica de la cantidad y tipo de recursos identificados a fin de determinar los patrones de variación del tipo de valoración realizada. Finalmente, se estableció una correlación entre el tipo de evaluación detectado y los fenómenos sociales evaluados. La combinación de estos análisis de cantidad de instancias de valoración y tipo de evaluación construida constituyen las bases para el análisis de la dinámica evaluativa de la problemática bajo análisis a lo largo de dos décadas.

\section{Resultados}

Los resultados de este estudio que presentamos aquí se despliegan en consonancia con el orden de los objetivos postulados. Primeramente, se exponen los hallazgos referidos a los recursos valorativos para la construcción de la valoración y luego se presentan los relativos a la dinámica evaluativa que esos recursos construyen a lo largo del tiempo.

Cabe aclarar que, en línea con la configuración típica del género discursivo noticias blandas, el dominio semántico predominante de la Actitud fue el compuesto por valores de Juicio, seguidos por Afecto y, muy cercanamente, de Apreciación, tal como se observa en el Gráfico 1.

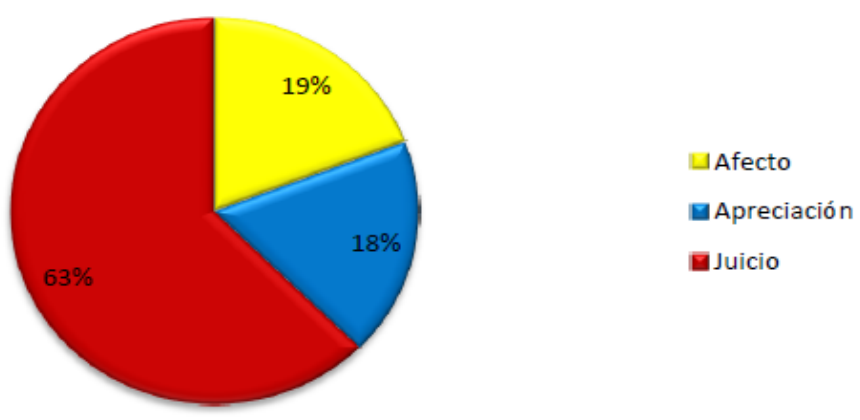

Gráfico 1. Composición de la Actitud expresada en porcentaje de significados evaluativos y según dominio semántico. 
Desarrollamos los resultados a continuación según el Sistema de Valoración, ACTITUD, con los subsistemas de Afecto, Apreciación y Juicio, respectivamente.

\subsection{Sobre los recursos evaluativos predominantes para la construcción de la ACTITUD}

\subsubsection{La codificación del Juicio}

Las entidades más representadas y evaluadas en el corpus fueron personas, sucesos, procesos y valores relacionados con la dictadura militar (1976-1983), incluyendo militares, miembros de las Fuerzas Armadas y fuerzas de seguridad, por una parte, y opositores al gobierno militar y desaparecidos, por otra.

Se observó una consistencia con la configuración del subgénero, 'noticias de interés humano’ y con la temática, en tanto los artículos reportan una temática controvertida y de profunda sensibilidad social. En consecuencia, los valores de Juicio fueron los predominantes, aun cuando el Afecto y la Apreciación también tuvieron su lugar de relativa relevancia. La Tabla 2 presenta los recursos léxico-gramaticales predominantes en la composición del Juicio y ejemplos extraídos del corpus ${ }^{1}$.

Tabla 2. Recursos léxico-gramaticales que codifican valores de Juicio, ejemplos y tipos de valores identificados.

\begin{tabular}{|c|c|c|}
\hline Ejemplo & $\begin{array}{c}\text { Realización léxico- } \\
\text { gramatical }\end{array}$ & Tipo de Juicio \\
\hline $\begin{array}{l}\text { Consignas de rechazo a la que fue la más cruel de las } \\
\text { tiranías que soporto el país (T29-LC) }\end{array}$ & $\begin{array}{l}\text { Epíteto en una } \\
\text { nominalización }\end{array}$ & $\begin{array}{l}\text { Sanción Social: } \\
\text { Integridad }\end{array}$ \\
\hline $\begin{array}{l}\text { Estas reglas, dijo Bonafini, son "anticonstitucionales" } \\
\text { (T5-ET) }\end{array}$ & $\begin{array}{l}\text { Valor con un proceso } \\
\text { relacional }\end{array}$ & $\begin{array}{l}\text { Sanción Social: } \\
\text { Integridad }\end{array}$ \\
\hline \multirow{2}{*}{$\begin{array}{l}\text { los genocidas continúan gozando de libertad (T29- } \\
\text { LC) }\end{array}$} & Nominalización & \multirow{2}{*}{$\begin{array}{l}\text { Sanción Social: } \\
\text { Integridad }\end{array}$} \\
\hline & Actores sociales & \\
\hline $\begin{array}{l}\text { Bonafini denunció "la corrupción del poder político } \\
\text { que negocia a espaldas del pueblo (T33-LN) }\end{array}$ & Carácter de los actores & $\begin{array}{l}\text { Sanción Social: } \\
\text { Integridad }\end{array}$ \\
\hline $\begin{array}{l}\text { ejercen todavía una nueva forma de tiranía que la } \\
\text { democracia no ha conseguido derrocar (T8-ET) }\end{array}$ & Procesos políticos & $\begin{array}{l}\text { Sanción Social: } \\
\text { Integridad }\end{array}$ \\
\hline ... ser encaminada hacia la reconciliación (T32-LN) & Procesos sociales & $\begin{array}{l}\text { Estima Social: } \\
\text { Tenacidad }\end{array}$ \\
\hline $\begin{array}{l}\text { Graciela Fernández Meijide criticó la medida del } \\
\text { gobierno de decidir la realización de un desfile militar } \\
\text { en momentos en que se desarrolla el juicio a los } \\
\text { excomandantes (T32-LN) }\end{array}$ & Proceso verbal & $\begin{array}{l}\text { Estima Social: } \\
\text { Tenacidad }\end{array}$ \\
\hline $\begin{array}{l}\text { no sabemos por qué no apresan a los otros } \\
\text { delincuentes (T1-ET) }\end{array}$ & Proceso mental & $\begin{array}{l}\text { Sanción Social: } \\
\text { Integridad }\end{array}$ \\
\hline reconocieron haber torturado a disidentes (T5-ET) & Proceso material & $\begin{array}{l}\text { Sanción Social: } \\
\text { Integridad }\end{array}$ \\
\hline $\begin{array}{l}\text {... la señora de Bonafini al calificar su actitud como } \\
\text { "una visita de cortesía" (T1-ET) }\end{array}$ & $\begin{array}{l}\text { Expresión idiomática - } \\
\text { Metáfora ideacional }\end{array}$ & $\begin{array}{l}\text { Estima Social: } \\
\text { Tenacidad }\end{array}$ \\
\hline
\end{tabular}

Según Martin y White (2005: 42), los valores de Juicio representan "las actitudes hacia la conducta que admiramos o criticamos, alabamos o condenamos". Resulta 
interesante destacar que la mayoría de los valores identificados fueron de tipo Sanción Social de la Integridad, consistentes con una temática que se encuentra en permanente tela de juicio por la naturaleza de las acciones que se juzgan, usualmente carentes de integridad, como lo son los actos no solo delictivos, desde una perspectiva estrictamente legal, sino inhumanos, cobardes y crueles, como puede observarse en los siguientes ejemplos:

(1) "La plaza es de las madres y no de los cobardes". (T12-LA)

(2) "Me guia la justicia y la lucha contra la impunidad". (T20-LA)

(3) Los genocidas continúan gozando de libertad (T29-LC)

Resulta oportuno recordar que el Juicio opera a modo de activación de ciertas creencias sobre "la manera en la cual deberían ser las cosas en la sociedad" (Feez et al., 2008: 151), lo cual corrobora la naturaleza estabilizadora de los discursos de medios, en particular los del tipo noticias blandas o de interés humano, tal lo presentado en el Gráfico 2.

\subsubsection{La codificación del Afecto}

En lo que respecta al Afecto, si bien este tipo de significados evaluativos no constituye la preocupación primordial del género noticias, resulta evidente que contribuye al tono valorativo general de los textos y, a su vez, opera estratégicamente en conjunción con los otros sistemas, marcando una impronta de cercanía o distanciamiento institucional de los medios productores de los textos con la temática.

Aunque se identificaron instancias explícitas de Afecto, en la mayoría de los casos los autores evocaban una reacción afectiva por medio de recursos que codifican los sentimientos y las emociones de modo implícito. Los ejemplos (14) a (19) ilustran la variedad y tipos de recursos utilizados para la expresión del Afecto en el corpus.

(4) Cuando 25 años no son nada (T8-ET)

(5) Una multitud marchó ... y rendir bomenaje de la peor dictadura que castigó a la Argentina. (T18-LA)

(6) ...no fueron pocos los que adbirieron al recuerdo de los desaparecidos con los rostros de emoción y satisfacción. (T28-LC)

(7) Hernán Schiller, ... negó que la presencia de aquellos núcleos hubiese sido irritativa... (T32-LN)

(8) Para el titular del Ejecutivo provincial, el país del futuro "se construye también sin odios y sin rencores". (T50-LV)

(9) Los manifestantes cantaron "Madres de la Plaza, el pueblo las abraza". (T53-RN)

Como puede observarse, el Afecto se codifica de modo directo, explícito, a través de procesos materiales como en el caso de (15), de procesos mentales como en (16) y (17), y mediante el uso de nominalizaciones como en los casos del ejemplo (18). En 
línea con las definiciones teóricas y analíticas realizadas, adoptamos la definición de nominalización de Halliday y Matthiessen (2014: 94), quienes la caracterizan como proceso por el cual "cualquier elemento o grupo de elementos opera como grupo nominal de la cláusula". Asimismo, vemos que el Afecto está evocado de modo implícito mediante metáforas como en (14), ejemplo en el que se construye la forma metafórica a partir del uso de un juego de palabras con la letra de un tango de alto contenido emotivo para la Argentina, Volver, de Carlos Gardel, o en el caso de (19), en el que el uso del proceso 'abrazar' es evidentemente metafórico, denotando un apoyo afectivo y empatía colectiva por el dolor sufrido.

\subsubsection{La codificación de la Apreciación}

La Apreciación es un dominio semántico que actúa para la "codificación de evaluaciones de tipo estético de textos, procesos y fenómenos naturales" (Martin \& White, 2005: 42). Si bien no es el valor predominante del corpus, constituye casi una quinta parte de las valoraciones identificadas. El tipo de Apreciación dominante fue el de Valuación. Al valuar un hecho, un proceso o un objeto, codificamos también implicancias positivas y negativas sobre la capacidad de los agentes creadores de esos entes, como se ilustra en el ejemplo a continuación.

(10) Elprimer hecho de anormalidad ocurrió minutos antes de las 11. (T1-ET)

(11) Severa critica al gobierno nacional por la crisis "que profundiza las desigualdades sociales". (T14-LA)

Como puede observarse en estos ejemplos, los valores identificados, codificados en su mayoría mediante cláusulas hipotácticas adjetivas, se hallan cercanos al plano del Juicio. Si bien no evalúan a los actores sociales de modo directo, codifican significados sobre fenómenos o procesos en términos de su relevancia social y de manera indirecta a los agentes sociales responsables por ellos.

En consonancia con el género discursivo, se tiende a evitar la realización por medio de recursos lexicales que pueden considerarse demasiado 'obvios' para una arena de pretendida objetividad como lo son las noticias, evitando epítetos u otras formas adjetivales, y optando por realizaciones de mayor elaboración sintáctica como la que aquí se ilustra.

De manera sucinta, podemos afirmar que este análisis demuestra que la ACTITUD se puede codificar tanto de manera explícita en las realizaciones léxico-gramaticales que pueden ser de una variedad de estructuras, como de forma invocada a través de huellas lingüísticas, usualmente metafóricas, que invitan al lector a alinearse con la posición evaluativa del medio. 


\subsection{Sobre la dinámica evaluativa}

El segundo objetivo planteado en este trabajo hace referencia a la manera en que las evaluaciones y las posturas valorativas van modificándose a lo largo del tiempo. Ya en 2002, Martin sugería que esta era una característica emergente de los análisis de discursos de reconciliación, en tanto su principal objetivo es la restauración, la reconstrucción del tejido social. La naturaleza del corpus nos permitió corroborar que esta idea sugerida décadas atrás es, en efecto, una característica distintiva de este discurso, de este género y en esta dimensión experiencial de situaciones de reconstrucción social post-traumática. En particular, el análisis de las instancias de ACTITUD demostró un predominio creciente de formas léxico-gramaticales que combinan sistemáticamente significados de Afecto y Juicio. Esto difiere parcialmente con lo planteado por Martin, quien proponía que las combinaciones se presentaban a nivel de valores de Afecto en conjunto con Apreciación.

En este estudio, esta fue una característica particular de la última etapa analizada. Esto parece indicar que la construcción de la memoria se desarrolla de manera gradual, una vez que se ha atravesado el período inicial de trauma profundo. A partir de 2000 en particular, se produce un quiebre en el tipo de valoraciones construidas en el discurso analizado.

Carlos Menem gobernó la Argentina ininterrumpidamente durante una década, en virtud de una reelección y una reforma de la Constitución Nacional en 1994 que le permitió permanecer por más tiempo en el gobierno. Fue representante del liberalismo acérrimo y negador sistemático de los intentos de reconstrucción de las relaciones sociales armónicas, de la lucha por develar la verdad sobre la triste historia vivida y un opositor constante de las asociaciones defensoras de derechos humanos. Una vez finalizados sus mandatos, la 'década perdida', como se la conoce popularmente, se observa un giro en las evaluaciones del pasado. Ya no son solamente Juicios, sino que gradualmente se comienzan a combinar con expresiones de Afecto. Los resultados indican que los argentinos pudieron en ese momento dar un paso adelante hacia la superación parcial del trauma, vinculándose con sus emociones y sentimientos, que hasta entonces estaban acallados. Resulta evidente que la ideología del gobierno de turno con respecto a la temática de la dictadura fue un factor determinante en estas configuraciones valorativas. Este tema es desarrollado en otros trabajos (véase Pascual, 2014, 2017a, 2017b, 2020, por ejemplo) que escapan a los límites del presente trabajo.

Los recursos lingüísticos por medio de los cuales se produjo este fenómeno de cambio de tipos de evaluaciones fueron principalmente de naturaleza léxica, realizados en procesos verbales o en participantes que introducen valoraciones de Afecto y Juicio, tanto positivas como negativas. A modo de ilustración, y como un breve 
glosario, la Tabla 3 presenta los procesos y participantes más frecuentes identificados en nuestro corpus.

Tabla 3. Procesos verbales y participantes que co-construyen significados valorativos de Afecto y Juicio en períodos de construcción de memoria colectiva.

\begin{tabular}{|l|l|}
\hline $\begin{array}{l}\text { Procesos indicadores de polaridad } \\
\text { positiva (+) }\end{array}$ & $\begin{array}{l}\text { apoyar, comprometer/se, exigir, instar a, reclamar, reivindicar, respaldar, } \\
\text { solicitar }\end{array}$ \\
\hline $\begin{array}{l}\text { Procesos indicadores de polaridad } \\
\text { negativa (-) }\end{array}$ & $\begin{array}{l}\text { acusar, advertir, condenar, criticar, denunciar, fustigar, impedir, negar, } \\
\text { pedirperdón, perdonar, pretender, rechazar, reprobar, reprochar, repudiar, } \\
\text { sancionar }\end{array}$ \\
\hline
\end{tabular}

La superposición de diversas capas de significado valorativo predominó en su polaridad negativa en términos cuantitativos de manera regular a lo largo de la última etapa del corpus, como se observa en la siguiente tabla (Tabla 4). Podemos observar que, si bien predominan las valoraciones negativas, y aunque prevalezca la cantidad de procesos al ser comparadas con las formas que cumplen una función de participantes, las cifras son elevadas en todos los casos. Tal es así, que las 270 instancias identificadas constituyen el $22 \%$ del total de las valoraciones identificadas en el corpus.

Tabla 4. Distribución de las instancias según su polaridad y función en el discurso.

\begin{tabular}{|c|c|c|c|c|}
\hline \multirow[t]{2}{*}{ Total de instancias } & \multicolumn{2}{|c|}{102 instancias $(+)$} & \multicolumn{2}{|c|}{168 instancias (-) } \\
\hline & 60 procesos & 42 participantes & 105 procesos & 63 participantes \\
\hline Total general & \multicolumn{4}{|c|}{270 instancias } \\
\hline
\end{tabular}

A modo de ejemplo de este fenómeno de superposición de significados, presentamos seguidamente el caso de 'repudiar', que registró 43 instancias, con sus diversas formas léxico-gramaticales. Como proceso:

(23) Nora Cortiñas, de Madres, afirmó que se repudiaba el golpe de Estado y también todo lo decidido actualmente en el Congreso nacional. (T48-LV)

Como participante en función de verbiage en clausulas verbales o atributo en clausulas materiales:

(24) Las Madres de Plaza de Mayo, al realizar su habitual marcha de los jueves, expresaron su repudio al desfile militar de ayer [...]. (T3-ET)

Como epíteto calificador del núcleo del participante:

(25) Los que bicieron este hecho tenebroso y macabro [...] tienen un solo nombre: son asesinos repudiados por el pueblo argentino", enfatizó Kirchner. (T30-LC) 
Estos ejemplos indican que la combinación de significados que operan en la construcción de diversos tipos de valoración se presenta en una variedad de formas lexicales y gramaticales.

De este modo, podemos afirmar que la Dinámica Evaluativa (DE) constituye un indicador discursivo del desarrollo de la construcción de la memoria colectiva. En el caso del corpus analizado, presentó las siguientes características:

- Predominio de valores de Juicio constante en todos los medios analizados, corroborando la configuración evaluativa del género, con presencia creciente hacia fines del período.

- Cuando Argentina salía de la dictadura militar e ingresaba a procesos de funcionamiento institucional democrático, casi la totalidad de esos casos eran de Juicio.

- Con el transcurso de los años, y con el ingreso de Menem al gobierno, y de su mano, de los modelos neoliberales, las voces de las agrupaciones defensoras de Derechos Humanos se silenciaron.

- Gradualmente, el discurso se fue construyendo sobre otros ejes experienciales. Dejó de reclamarse por la aparición con vida de los desaparecidos. La sociedad comenzó gradual y dolorosamente a asumir que los desaparecidos eran muertos.

- Desde mediados de los 90, el reclamo social de Justicia, de Verdad, que comenzó a codificar valores de Juicio que se fusionaban con los de Afecto: la necesidad de Memoria, de que los argentinos no se olvidaran de quienes habían desaparecido.

- En línea con estos cambios sociohistóricos, se transformó la configuración de la evaluación, con valores que cada vez se acercaban más a la combinación de estrategias de Juicio y Afecto, y que construyeron discursivamente nociones dinámicas del dolor, la vergüenza, la reconciliación y el homenaje.

Estas características se hallan en línea con las afirmaciones de Assman (2009) sobre la construcción discursiva de lo que esta autora denomina Memoria para olvidar, una de las formas de la memoria colectiva que se caracteriza por su capacidad terapéutica, en la cual las sociedades rompen el silencio para poder superar el dolor, la culpa y el odio por las atrocidades cometidas. Esta forma de memoria constituye un requisito indispensable para superar la experiencia traumática vivida y poder enfrentar una idea de futuro colectivo de convivencia relativamente armoniosa.

\section{CONCLUSIONES}

Los resultados de este trabajo dan cuenta de una mutación en la construcción en el eje de los significados que codifican Juicios, tanto de sanción como de estima social. Resulta interesante destacar que a medida que se aleja el hecho social traumático de la 
dictadura, se generan combinaciones de significados que incorporan elementos de Afecto. Postulamos que estos significados se fusionan para recontextualizar discursivamente cómo la sociedad enfrentó su pasado reciente y desarrolló su memoria colectiva. En línea con estos hallazgos, se pudo observar que la predominancia de Juicios explícitos dio lugar a lo largo de los años a valores de Afecto explícito que invocan Juicios.

Asimismo, los datos analizados permitieron sistematizar los recursos valorativos utilizados y proponer un glosario de lexemas relacionados con las dimensiones evaluativas de los discursos sobre pasados traumáticos, que en el futuro puede ser extendido y refinado en su nivel de detalle, al ser contrastado con otros estudios de situaciones post-traumáticas. La propuesta también resulta de utilidad en tanto permite identificar, sistematizar e interpretar la construcción discursiva de fenómenos sociales de profundo impacto traumático, como las guerras civiles, dictaduras, invasiones, enfrentamientos religiosos y muchos otros conflictos sociales que dejan profundas huellas de dolor en nuestras sociedades.

Muchos de estos fenómenos han sido descriptos en la bibliografía. Sin embargo, el enfoque particular que adoptamos desde el Sistema de la Valoración nos brinda valiosos aportes en tanto viabiliza la definición de un fenómeno semántico discursivo particular, la Dinámica Evaluativa, y la caracterización en términos de la LSF de un fenómeno social de permanente actualidad y relevancia: los conflictos sociales y cómo reaccionamos ante ellos.

\section{REFERENCIAS BIBLIOGRÁFICAS}

Achugar, M. (2008). What we remember: The construction of military memory in military discourse. Ámsterdam: John Benjamins.

Assmann, A. (2009). From collective violence to a common future: Four models for dealing with a traumatic past. En R. Wodak \& G. Auer (Eds.), Memory and Justice (pp. 31-48). Viena: Passagen Verlag.

Benítez, D. H. \& Mónaco, C. (2007). La dictadura militar, 1976-1983. En G. Kessler \& M. Luzzi (Comps.), Problemas socioeconómicos contemporáneos. Los Polvorines: Universidad Nacional de General Sarmiento [en línea]. Disponible en: http://www.riehr.com.ar/archivos/Educacion/La\%20dictadura\%20militar $\% 20$ Monaco $\% 20$ Benitez.pdf

Fairclough, N. (2010). Critical discourse analysis: The critical study of language. Londres: Routledge.

Feez, S., Iedema, R. \& White, P. R. R. (2008). Media literacy. Sydney: NSW Adult Migrant Education Service. 
Halliday, M. A. K. (1978). Language as a social semiotic. Londres: Edward Arnold.

Halliday, M. A. K. \& Matthiessen, C. M. I. M. (2014). An introduction to functional grammar. Fourth edition. Londres/Nueva York: Routledge.

Hood, S. (2005). What is evaluated and how in academic research writing? The copatterning of attitude and field. Australian Review of Applied Linguistics Series. S(19), 23-40.

Hood, S. \& Martin, J. R. (2005). Invocación de actitudes: El juego de la gradación de la valoración en el discurso. Revista Signos. Estudios de Lingüística, 38(58), $195-$ 220.

Martin, J. R. (2000). Beyond exchange: Appraisal systems in English. En S. Hunston \& G. Thompson (Eds.), Evaluation in Text: Authorial Stance and the Construction of Discourse (pp.142-175). Oxford: OUP.

Martin, J. R. (2002). Blessed are the peacemakers: Reconciliation and evaluation. En C. Candlin (Ed.), Research and Practice in Professional Discourse (pp.187-227). Hong Kong: City University of Hong Kong Press.

Martin, J. R. (2017). Attitudinal relations: Continuing the study of lexis. En L. Barbara, A. S. Rodriques-Júnior \& G. M. V. Hoy (Eds.), Estudos e Pesquisas em Linguística Sistêmico-Funcional (pp. 53-87). São Paulo: Mercado de Letras.

Martin, J. R. \& Rose, D. (2008). Genre relations: Mapping culture. Londres: Equinox.

Martin, J. R. \& White, P. R. R. (2005). The Language of evaluation: Appraisal in English. Londres/Nueva York: Palgrave/Macmillan.

Oteíza, T. (2010). Patrones valorativos en el discurso oficial de Derechos Humanos en Chile: Dando valor al pasado y construyendo memorias históricas en la Sociedad. Discurso y Sociedad, 4(1), 151-183.

Pascual, M. (2014). La asociación de recursos de valoración: El caso de las organizaciones de derechos humanos en la prensa escrita argentina. Onomázein 1(29) 99-114.

Pascual, M. (2017a). Coming to terms with a traumatic past: Social actors in the Argentine media. En S. Bemposta-Rivas, C. Bouzada-Jabois, Y. FernándezPena, T. Bouso, Y. J. Calvo-Benzies \& I. Tamaredo (Eds.), New trends and methodologies in applied English language research III (pp. 207-231). Bern/Nueva York: Peter Lang. 
Pascual, M. (2017b). La violación a los derechos humanos en la Argentina: Dinámica evaluativa de dos décadas de representación en medios. Discurso \& Sociedad, 11(3), 388-413.

Pascual, M. (2020). El papel del Estado en la construcción de la memoria: El discurso de medios sobre el Museo de la Memoria de Buenos Aires. Estudios del Discurso, 5(2) 66-88.

Reisigl. M. \& Wodak, R. (2001). Discourse and discrimination: Rhetorics of racism and antisemitism. Londres, UK: Routledge.

van Dijk, T.A. (2008). Discourse and power. Houndsmills: Palgrave.

White, P. R. R. (2002). Appraisal: The language of evaluation and stance. En J. Verschueren, J. O. Östman, J. Blommaert \& Chris Bulcaen (Eds.), Handbook of Pragmatics (pp.1-27). Ámsterdam: John Benjamins.

White, P. R. R. (2003). Beyond modality and hedging: A dialogic view of the language of intersubjective stance. Text - Special Edition on Appraisal, 259-284.

Wodak, R. (1995). Critical linguistics and critical discourse analysis. En J. O. Varscheueren, J-O Östman \& J. Blommart (Eds.), Handbook of Pragmatics (pp. 204-210). Ámsterdam: John Benjamins.

Wodak, R. (1996). Disorders of discourse. Londres: Longman.

Wodak, R. (2007). Pragmatics and critical discourse analysis. A cross-disciplinary enquiry. Pragmatics \& Cognition, 15(1), 203-225.

Wodak, R. (Ed). (2013). Critical discourse analysis. Londres, UK: Sage.

Wodak, R. \& Meyer, M. (Eds.) (2009). Methods for critical discourse analysis. Londres: Sage.

\section{NOTA}

${ }^{1}$ Los textos del corpus se identifican con número de codificación numérica (texto $1=\mathrm{T} 1$; texto $2=\mathrm{T} 2$, etc.) junto con el código correspondiente al medio del cual se extrajo, con las siglas de su denominación; a saber: El Tribuno= ET; Los Andes=LA; La Capital= LC; La Nación=LN; La Voz del Interior=LV y Río Negro= RN. De esta manera, por ejemplo, T29-LC corresponde al texto 29, extraído del diario La Capital. 